\title{
Dynamics of extended spinning masses in a gravitational field
}

\author{
Bahram Mashhoon* \\ Department of Physics and Astronomy, University of Missouri-Columbia, Columbia, Missouri, 65211, USA
}

Dinesh Singh $^{\dagger}$

Department of Physics, University of Regina, Regina, Saskatchewan, S4S 0A2, Canada

(Received 15 August 2006; published 6 December 2006)

\begin{abstract}
We develop a first-order approximation method for the influence of spin on the motion of extended spinning test masses in a gravitational field. This approach is illustrated for approximately circular equatorial motion in the exterior Kerr spacetime. In this case, the analytic results for the first-order approximation are compared to the numerical integration of the exact system and the limitations of the first-order results are pointed out. Furthermore, we employ our analytic results to illustrate the gravitomagnetic clock effect for spinning particles.
\end{abstract}

DOI: 10.1103/PhysRevD.74.124006

PACS numbers: $04.20 . \mathrm{Cv}$

\section{INTRODUCTION}

Imagine the motion of an extended spinning body in the exterior vacuum region of an astronomical source. Let $T^{\mu \nu}$ be the energy-momentum of the extended test mass; then, the motion is governed by the four equations that are given by the dynamical law $T_{; \nu}^{\mu \nu}=0$. Consider a representative point inside the extended test body (the "center of mass") such that $u_{s}^{\mu}=d x^{\mu} / d \sigma$ is its four-velocity vector and $\sigma$ is the proper time along its worldline. The equations of motion can then be expressed relative to the chosen worldline. This has been accomplished in an elegant manner by Dixon [1]. Using Synge's world function [2], Dixon has defined the infinite set of multipole moments of $T_{\mu \nu}$ in a way that is qualitatively similar to the standard nonrelativistic theory and thereby expressed the equations of motion as [1]

$$
\begin{aligned}
& \frac{D P^{\mu}}{d \sigma}=-\frac{1}{2} R_{\nu \alpha \beta}^{\mu} u_{s}^{\nu} S^{\alpha \beta}+\mathcal{F}^{\mu}, \\
& \frac{D S^{\mu \nu}}{d \sigma}=P^{\mu} u_{s}^{\nu}-P^{\nu} u_{s}^{\mu}+\mathcal{T}^{\mu \nu} .
\end{aligned}
$$

Here, the momentum vector $P^{\mu}$ and the spin tensor $S^{\mu \nu}$ are the first two moments of $T^{\mu \nu}$, while the Dixon force $\mathcal{F}^{\mu}$ and torque $\mathcal{T}^{\mu \nu}$ are given in terms of the quadrupole and higher moments of the test body. It is necessary to add a supplementary condition to Eqs. (1) and (2) in order to fix an appropriate center-of-mass trajectory. It turns out that for an extended body the appropriate condition is [3]

$$
S^{\mu \nu} P_{\nu}=0 .
$$

The work of Dixon provides a natural generalization of the previous results of Mathisson [4] and Papapetrou [5] that were limited to pole-dipole particles.

\footnotetext{
*Electronic address: mashhoonb@missouri.edu

${ }^{\dagger}$ Electronic address: singhd@uregina.ca
}

In the present paper, we are interested in the dynamics of an extended body of mass $m$ and spin $s$ in the field of a rotating mass $M \gg m$; therefore, we consider Eqs. (1)-(3) without the quadrupole and higher moments, i.e., $\mathcal{F}^{\mu}=0$ and $\mathcal{T}^{\mu \nu}=0$. The resulting reduced MathissonPapapetrou-Dixon (MPD) equations then lead to natural definitions for the mass $m$ and spin $s$ of the extended test particle that are preserved throughout the motion. We assume that the metric tensor has signature +2 and we use units such that $c=G=1$, unless otherwise specified. It then follows from Eqs. (1)-(3) that in this case the mass of the extended particle is given by $m=\left(-P^{\mu} P_{\mu}\right)^{1 / 2}$, which is a constant of the motion. The spin vector is then defined by

$$
S_{\mu}=-\frac{1}{2 m} \epsilon_{\mu \nu \rho \sigma} P^{\nu} S^{\rho \sigma}, \quad S^{\mu \nu}=\frac{1}{m} \epsilon^{\mu \nu \rho \sigma} P_{\rho} S_{\sigma},
$$

where $\epsilon_{\mu \nu \rho \sigma}$ is the Levi-Civita tensor given by $\epsilon_{\mu \nu \rho \sigma}=$ $\sqrt{-g} \sigma_{\mu \nu \rho \sigma}$ in terms of the alternating symbol with $\sigma_{0123}=1$. It follows from the reduced MPD equations that $s^{2}=S_{\mu} S^{\mu}=\frac{1}{2} S_{\mu \nu} S^{\mu \nu}$ is a constant of the motion. Moreover, the requirement that the dipole force be much smaller than the monopole force implies that $s \ll m r$, where $r$ is the distance between $m$ and $M$; that is, the Møller radius of the extended test particle $(\mathrm{s} / \mathrm{m})$ must be very small compared to its distance from the source.

In a recent paper [6], an approximation scheme has been introduced to deal with this extended pole-dipole system in most astrophysical situations. This scheme is based on the circumstance that $m^{-1} P^{\mu}-u_{s}^{\mu}$ is small and of order $(M / r)[s /(m r)]^{2} \ll 1$. In this approach, it then turns out that to first order in $s /(m r)$ one can assume that $P^{\mu}$ and $u_{s}^{\mu}$ are parallel, namely, $P^{\mu} \approx m u_{s}^{\mu}$; therefore, it follows from Eqs. (1)-(3) in this case that

$$
\begin{gathered}
\frac{D u_{s}^{\mu}}{d \sigma} \approx-\frac{1}{2 m} R_{\nu \alpha \beta}^{\mu} u_{s}^{\nu} S^{\alpha \beta}, \quad \frac{D S^{\mu \nu}}{d \sigma} \approx 0, \\
S_{\mu \nu} u_{s}^{\nu} \approx 0 .
\end{gathered}
$$


The second section of Ref. [6] should be consulted for the details of the derivation of these equations.

In the absence of spin, system (5) simply reduces to the geodesic equation for the motion of the center of mass of the spinless extended particle, as expected [7]. In Sec. II, we solve system (5) assuming that in the zeroth order of approximation the worldline for a spinless particle is a timelike geodesic. The motion away from the geodesic is then of first order in $s /(m r)$ in our approach. This method is employed to study approximately circular motion near the equatorial plane of a Kerr source of mass $M$ and angular momentum $J=M a$ in Sec. III. That is, we assume that in the absence of spin, the test mass follows a stable equatorial circular geodesic orbit in the exterior Kerr spacetime. Our approximate analytic results based on system (5) are compared with the numerical solution of the extended pole-dipole system, Eqs. (1)-(3), in Kerr spacetime in Sec. IV. Section V is devoted to the gravitomagnetic clock effect. A brief discussion of our results is contained in Sec. VI. The appendices contain further developments of our main results.

\section{FIRST-ORDER APPROXIMATION}

Consider the motion of an extended spinning test particle in a gravitational field. Let $u^{\mu}$ be the four-velocity of the particle in the absence of spin and $\lambda^{\mu}{ }_{\hat{\alpha}}$ be the orthonormal tetrad frame that is parallel propagated along the reference geodesic $x^{\mu}(\tau)$, where $u^{\mu}=d x^{\mu} / d \tau$ and $\tau$ is the proper time of the spinless particle. More specifically, we imagine that along the unperturbed reference geodesic worldline $x^{\mu}(\tau)$, the Mathisson-Papapetrou spin-curvature interaction is "turned on" at $\tau=0$ and the spinning particle then follows $x_{s}^{\mu}(\tau)$ for $\tau>0$. Let $\delta x^{\mu}(\tau)=x_{s}^{\mu}(\tau)-$ $x^{\mu}(\tau)$ be the deviation between the neighboring worldlines at the same time $\tau>0$ such that $\left(\delta x^{\mu}\right) u_{\mu}=0$. We will work to linear order in the small quantity $s /(m r) \ll 1$. Here $\delta x^{\mu}(\tau)$ is a vector field defined orthogonally along $x^{\mu}(\tau)$ such that it connects the reference geodesic to the path of the spinning particle in a way that amounts to a unique identification of events along the perturbed trajectory using the proper time of the geodesic worldline $x^{\mu}$. It follows that we can write

$$
\delta x^{\mu}=X^{i}(\tau) \lambda^{\mu}{ }_{\hat{i}}
$$

where $X(\tau)$ can be determined by the equations of motion (5). To this end, we establish a Fermi normal coordinate system along $x^{\mu}(\tau)$ based on the local frame $\lambda^{\mu}{ }_{\hat{\alpha}}$ and solve the equations of motion in this Fermi coordinate system. The procedure that we follow is described in detail in Appendix A. Here we provide a general summary of our approximation method.

To linear order in our approximation scheme, it follows from Eq. (5) that the spin tensor $S^{\mu \nu}$ is parallel propagated along the unperturbed trajectory and $S_{\mu \nu} u^{\nu}=0$. One can then write

$$
S^{\mu \nu}=\lambda^{\mu}{ }_{i} \lambda_{\hat{\jmath}}^{\nu} S^{\hat{\imath} \hat{\jmath}},
$$

where $S^{\hat{\imath} \hat{\jmath}}$ are constants of the motion. Moreover, it is simple to show that in the linear approximation scheme under consideration in this paper, $S_{\mu} u^{\mu}=0$, so that for $S_{\hat{\alpha}}=S_{\mu} \lambda_{\hat{\alpha}}^{\mu}$ we have $S_{\hat{0}}=0$ and

$$
S_{\hat{\imath}}=\frac{1}{2} \epsilon_{i j k} S^{\hat{\jmath} \hat{k}} .
$$

Here, we have used the fact that $\epsilon_{\mu \nu \rho \sigma} \lambda^{\mu}{ }_{0} \lambda^{\nu}{ }_{1} \lambda^{\rho}{ }_{\hat{2}} \lambda^{\sigma}{ }_{\hat{3}}=1$, since $\epsilon_{\hat{0} \hat{1} \hat{z} \hat{z}}=1$; hence, $\operatorname{det}\left(\lambda_{\hat{\alpha}}^{\mu}\right)=(-g)^{-1 / 2}$, which is a consequence of the orthonormality of the tetrad frame.

Consider next the components of the spacetime curvature tensor measured by a free observer moving along the reference geodesic. These components, $R_{\mu \nu \rho \sigma} \lambda^{\mu}{ }_{\hat{\alpha}} \lambda_{\hat{\beta}}^{\nu} \lambda_{\hat{\gamma}}^{\rho} \lambda_{\hat{\delta}}^{\sigma}$, can be expressed as a $6 \times 6$ matrix $\left(\mathcal{R}_{\Delta \Sigma}\right)$, where the indices $\Delta$ and $\Sigma$ range over the set $\{01$, $02,03,23,31,12\}$. One can then write

$$
\mathcal{R}=\left(\begin{array}{cc}
\mathcal{E} & \mathcal{H} \\
\mathcal{H}^{\dagger} & \mathcal{D}
\end{array}\right)
$$

where $\mathcal{E}, \mathcal{H}$, and $\mathcal{D}$ are $3 \times 3$ matrices representing, respectively, the electric, magnetic, and spatial components of the Riemannian curvature. In general, $\mathcal{E}$ and $\mathcal{D}$ are symmetric and $\mathcal{H}$ is traceless. In a background Ricciflat spacetime, $\mathcal{D}=-\mathcal{E}, \mathcal{E}$ is traceless and $\mathcal{H}$ is symmetric $\left(\mathcal{H}=\mathcal{H}^{\dagger}\right)$. In this case, the curvature is completely determined by its electric and magnetic components. These are given in general by

$$
\begin{gathered}
\mathcal{E}_{\hat{\imath} \hat{\jmath}}=R_{\mu \nu \rho \sigma} \lambda^{\mu}{ }_{0} \lambda_{{ }_{i}}^{\nu} \lambda^{\rho}{ }_{\hat{0}} \lambda^{\sigma}{ }_{\hat{\jmath}}, \\
\mathcal{H}_{\hat{\imath} \hat{\jmath}}=\frac{1}{2} \epsilon_{j}{ }^{k l} R_{\mu \nu \rho \sigma} \lambda^{\mu}{ }_{\hat{0}} \lambda^{\nu}{ }_{\hat{\imath}} \lambda^{\rho}{ }_{\hat{k}} \lambda^{\sigma}{ }_{\hat{\imath}}{ }^{\prime}
\end{gathered}
$$

and, as described below, play important roles in our approximation scheme.

It is shown in Appendix A that the worldline of the spinning particle is given, in our linear approximation, by

$$
x_{s}^{\mu}(\tau)=x^{\mu}(\tau)+X^{i}(\tau) \lambda_{\hat{i}}^{\mu},
$$

where $X(\tau)$ is a solution of the system

$$
\begin{gathered}
\frac{d X^{i}}{d \tau}=V^{i}, \\
\frac{d V^{i}}{d \tau}+\mathcal{E}_{\hat{\imath} \hat{\jmath}}(\tau) X^{j}=\frac{1}{m} \mathcal{H}_{\hat{\imath} \hat{\jmath}}(\tau) S^{\hat{\jmath}},
\end{gathered}
$$

with the boundary conditions that at $\tau=0, X(0)=0$, and $\boldsymbol{V}(0)=0$. The unique solution of this system is thus due to the spin-curvature force folded together with the tidal influence of the background gravitational field. In fact, system (13) and (14) can be solved with the method of variation of parameters [8] once the fundamental solution of the homogeneous part is available. In the absence of spin, the system simply reduces to the Jacobi equation in the Fermi coordinate system; therefore, system (13) and 
(14) can be solved once the Jacobi fields along the path of the reference geodesic are completely known. The general solution of system (13) and (14) is discussed in Appendix A. In the following section, we apply this method to Kerr spacetime; that is, we solve Eqs. (13) and (14) for the case of a spinning test particle that follows an approximately circular equatorial orbit in the exterior Kerr field. The Kerr field, which represents the exact gravitational field of a rotating black hole, has been chosen here for the sake of simplicity; in principle, one could employ any other exact solution of the gravitational field equations describing the exterior field of a rotating mass [9].

\section{KERR SPACETIME}

Consider circular geodesic orbits about a Kerr source of mass $M$ and angular momentum $J$. In standard BoyerLindquist coordinates $(t, r, \theta, \phi)$, the geodesic equation for a circular equatorial orbit of fixed "radius" $r>2 M$ and $\theta=\pi / 2$ reduces to

$$
t=\frac{1}{N}\left(1+a \Omega_{\mathrm{K}}\right) \tau, \quad \phi=\frac{1}{N} \Omega_{\mathrm{K}} \tau,
$$

where $a=J / M>0$ and we have chosen boundary conditions such that $t=\phi=0$ at $\tau=0$,

$$
N=\sqrt{1-\frac{3 M}{r}+2 a \Omega_{\mathrm{K}}}
$$

and

$$
\Omega_{\mathrm{K}}= \pm \sqrt{\frac{M}{r^{3}}}
$$

is the Keplerian frequency of the orbit. Here the upper sign indicates an orbit that rotates in the same sense as the source, while the lower sign indicates a counterrotating orbit. The energy $E$ and orbital angular momentum $L$ associated with these circular orbits are given by

$$
\begin{aligned}
& E=\frac{1}{N}\left(1-\frac{2 M}{r}+a \Omega_{\mathrm{K}}\right), \\
& L=\frac{r^{2} \Omega_{\mathrm{K}}}{N}\left(1-2 a \Omega_{\mathrm{K}}+\frac{a^{2}}{r^{2}}\right) .
\end{aligned}
$$

A detailed discussion of Kerr geodesics can be found in Refs. [10,11] and references therein. Furthermore, the motion of spinning test particles in Kerr and other astrophysically interesting spacetimes has been studied by a number of authors using other approaches, as can be seen from a perusal of Refs. [12-23].

It is possible to set up an orthonormal parallelpropagated frame $\lambda_{\hat{\alpha}}^{\mu}$ along the geodesic worldline of the spinless test particle such that $\lambda^{\mu}{ }_{\hat{0}}=d x^{\mu} / d \tau$ is the particle's four-velocity vector and $\lambda^{\mu}{ }_{i}, i=1,2,3$, are unit gyro axes that form the particle's local spatial frame. It follows that in $(t, r, \theta, \phi)$ coordinates [24]

$$
\begin{gathered}
\lambda_{\hat{0}}^{\mu}=\left(\frac{1+a \Omega_{\mathrm{K}}}{N}, 0,0, \frac{\Omega_{\mathrm{K}}}{N}\right), \\
\lambda_{\hat{1}}^{\mu}=\left(-\frac{L}{r A} \sin \Theta, A \cos \Theta, 0,-\frac{E}{r A} \sin \Theta\right), \\
\lambda_{\hat{2}}^{\mu}=\left(0,0, \frac{1}{r}, 0\right), \\
\lambda_{\hat{\mathrm{J}}}^{\mu}=\left(\frac{L}{r A} \cos \Theta, A \sin \Theta, 0, \frac{E}{r A} \cos \Theta\right),
\end{gathered}
$$

where $\Theta=\Omega_{\mathrm{K}} \tau$ and $A$ is given by

$$
A=\sqrt{1-\frac{2 M}{r}+\frac{a^{2}}{r^{2}}}
$$

Furthermore, the electric and magnetic components of the spacetime curvature are given by [24]

$$
\mathcal{E}=\left(\begin{array}{ccc}
k_{1} & 0 & k^{\prime} \\
0 & k_{2} & 0 \\
k^{\prime} & 0 & k_{3}
\end{array}\right), \quad \mathcal{H}=\left(\begin{array}{ccc}
0 & h & 0 \\
h & 0 & h^{\prime} \\
0 & h^{\prime} & 0
\end{array}\right),
$$

where $k_{2}=-\left(k_{1}+k_{3}\right)$ is a constant given by $k_{2}=$ $k\left(3 \gamma^{2}-2\right)$ and

$$
\begin{gathered}
k_{1}=k\left(1-3 \gamma^{2} \cos ^{2} \Theta\right), \\
k_{3}=k\left(1-3 \gamma^{2} \sin ^{2} \Theta\right), \\
k^{\prime}=-3 \gamma^{2} k \sin \Theta \cos \Theta, \\
h=-3 \gamma^{2} \beta k \cos \Theta, \quad h^{\prime}=-3 \gamma^{2} \beta k \sin \Theta .
\end{gathered}
$$

Here

$$
k=\frac{M}{r^{3}}=\Omega_{\mathrm{K}}^{2},
$$

while $\beta$ and $\gamma$ constitute a Lorentz pair, $\gamma=1 / \sqrt{1-\beta^{2}}$, given by

$$
\beta=\frac{1}{A}\left(r \Omega_{\mathrm{K}}-\frac{a}{r}\right), \quad \gamma=\frac{A}{N} .
$$

The results presented here for the electric components of the curvature tensor are consistent with the work of Marck [25].

We can now employ these results in order to find the perturbed orbit $x_{s}^{\mu}(\tau)=\left(t_{s}, r_{s}, \theta_{s}, \phi_{s}\right)$ given by Eq. (12), namely,

$$
\begin{gathered}
t_{s}=\frac{1+a \Omega_{\mathrm{K}}}{N} \tau+\frac{L}{r A}(-X \sin \Theta+Z \cos \Theta), \\
r_{s}=r+A(X \cos \Theta+Z \sin \Theta), \\
\theta_{s}=\frac{\pi}{2}+\frac{Y}{r}
\end{gathered}
$$




$$
\phi_{s}=\frac{\Omega_{\mathrm{K}}}{N} \tau+\frac{E}{r A}(-X \sin \Theta+Z \cos \Theta),
$$

where $\boldsymbol{X}=(X, Y, Z)$ and the tetrad frame given by Eqs. (19)-(22) has been used. It is useful to write $S_{\hat{\imath}}$ in terms of spherical polar coordinates $(s, \vartheta, \varphi)$ with respect to the local tetrad frame as

$$
\begin{gathered}
S_{\hat{1}}=s \sin \vartheta \cos \varphi, \quad S_{\hat{2}}=-s \cos \vartheta, \\
S_{\hat{\mathrm{Z}}}=s \sin \vartheta \sin \varphi,
\end{gathered}
$$

so that $s \cos \vartheta$ is the component of the spin vector along the rotation axis of the Kerr source. Then system (13) and (14) takes the form

$$
\begin{aligned}
\frac{d^{2} X}{d \Theta^{2}}= & \left(3 \gamma^{2} \cos ^{2} \Theta-1\right) X+3 \gamma^{2} \sin \Theta \cos \Theta Z \\
& +3 \gamma^{2} \beta\left(\frac{s}{m} \cos \vartheta\right) \cos \Theta \\
\frac{d^{2} Y}{d \Theta^{2}}= & \left(2-3 \gamma^{2}\right) Y-3 \gamma^{2} \beta\left(\frac{s}{m} \sin \vartheta\right) \cos (\Theta-\varphi) \\
\frac{d^{2} Z}{d \Theta^{2}}= & 3 \gamma^{2} \sin \Theta \cos \Theta X+\left(3 \gamma^{2} \sin ^{2} \Theta-1\right) Z \\
& +3 \gamma^{2} \beta\left(\frac{s}{m} \cos \vartheta\right) \sin \Theta
\end{aligned}
$$

with the boundary conditions that $\boldsymbol{X}=\mathbf{0}$ and $\dot{\boldsymbol{X}}=\mathbf{0}$ at $\Theta=0$. Here an overdot denotes differentiation with respect to $\Theta$. Let us now consider a rotation about the vertical axis by an angle $\Theta$ so that the rotated coordinate axes correspond to the radial, vertical, and tangential directions; that is, $Y^{\prime}=Y$ and

$$
X^{\prime}=X \cos \Theta+Z \sin \Theta, \quad Z^{\prime}=-X \sin \Theta+Z \cos \Theta .
$$

We note that $x_{s}^{\mu}$ can be conveniently expressed in terms of the rotated coordinates $\left(X^{\prime}, Y^{\prime}, Z^{\prime}\right)$, as they appear explicitly in Eqs. (29)-(32) for the perturbed orbit.

It is possible to show that under rotation (37), Eqs. (34)(36) take the form

$$
\begin{gathered}
\ddot{X}^{\prime}-2 \dot{Z}^{\prime}-3 \gamma^{2} X^{\prime}=3 \gamma^{2} \beta\left(\frac{s}{m} \cos \vartheta\right) \\
\ddot{Y}^{\prime}+\left(3 \gamma^{2}-2\right) Y^{\prime}=-3 \gamma^{2} \beta\left(\frac{s}{m} \sin \vartheta\right) \cos (\Theta-\varphi) \\
\ddot{Z}^{\prime}+2 \dot{X}^{\prime}=0
\end{gathered}
$$

with the boundary conditions that at $\Theta=0, \boldsymbol{X}^{\prime}=\mathbf{0}$, and $\dot{\boldsymbol{X}}^{\prime}=\mathbf{0}$. The unique solution of this system is given by

$$
X^{\prime}=\frac{3}{\rho^{2}} \gamma^{2} \beta\left(\frac{s}{m} \cos \vartheta\right)[1-\cos (\rho \Theta)],
$$

$$
\begin{aligned}
Y^{\prime}= & \frac{1}{\beta}\left(\frac{s}{m} \sin \vartheta\right)\left[\cos \varphi \cos (\zeta \Theta)+\frac{1}{\zeta} \sin \varphi \sin (\zeta \Theta)\right. \\
& -\cos (\Theta-\varphi)], \\
Z^{\prime} & =-\frac{6}{\rho^{3}} \gamma^{2} \beta\left(\frac{s}{m} \cos \vartheta\right)[\rho \Theta-\sin (\rho \Theta)] .
\end{aligned}
$$

Here

$$
\rho=\sqrt{4-3 \gamma^{2}}, \quad \zeta=\sqrt{3 \gamma^{2}-2}
$$

are such that $\rho\left|\Omega_{\mathrm{K}}\right|$ and $\zeta\left|\Omega_{\mathrm{K}}\right|$ are the proper radial and vertical epicyclic frequencies, respectively.

It follows from these results that the orbit of the spinning particle in the standard Kerr coordinate system is given by

$$
t_{\mathrm{s}}=\frac{1+a \Omega_{\mathrm{K}}}{N} \tau+\frac{6}{\rho^{3}} \gamma^{2} \beta\left(\frac{s}{m r} \cos \vartheta\right) \frac{L}{A}[\sin (\rho \Theta)-\rho \Theta],
$$

$$
\begin{aligned}
r_{\mathrm{s}}=r\{1+ & \left.\frac{3}{\rho^{2}} \gamma^{2} \beta\left(\frac{s}{m r} \cos \vartheta\right) A[1-\cos (\rho \Theta)]\right\}, \\
\theta_{\mathrm{s}}= & \frac{\pi}{2}+\frac{1}{\beta}\left(\frac{s}{m r} \sin \vartheta\right)[\cos \varphi \cos (\zeta \Theta) \\
& \left.+\frac{1}{\zeta} \sin \varphi \sin (\zeta \Theta)-\cos (\Theta-\varphi)\right],
\end{aligned}
$$

$$
\phi_{\mathrm{s}}=\frac{\Omega_{\mathrm{K}}}{N} \tau+\frac{6}{\rho^{3}} \gamma^{2} \beta\left(\frac{s}{m r} \cos \vartheta\right) \frac{E}{A}[\sin (\rho \Theta)-\rho \Theta] .
$$

The perturbation in $\phi_{s}$ is simply $E / L$ times the perturbation in $t_{s}$. In Eqs. (45)-(48), the temporal parameter $\tau$ can be replaced by $\sigma$, which is the proper time of the perturbed worldline, since $\tau=\sigma+O\left(s^{2}\right)$ by Eqs. (A7)-(A9) of Appendix A. It is interesting to note here the role of spin length scales

$$
s^{*}=\left(\frac{s}{m}\right) \cos \vartheta, \quad \tilde{s}=\left(\frac{s}{m}\right) \sin \vartheta
$$

in the worldline of the particle; the perturbations in $t_{s}, r_{s}$, 
and $\phi_{s}$ are proportional to $s^{*}$, while the polar motion away from the equatorial plane is proportional to $\tilde{s}$.

Equations (45)-(48), involving the first-order perturbation terms proportional to $s /(m r)$, should be viewed as containing the zeroth and first-order terms of a perturbation expansion in powers of a sufficiently small parameter $s /(m r) \ll 1$. Moreover, for a black hole $(a \leq M), \beta$ in Eqs. (45)-(48) is positive for corotating orbits and negative for counterrotating orbits, while $\gamma^{2}$ monotonically decreases from $4 / 3$ at the last stable circular orbit to unity at infinity. Similarly, $|\beta|$ decreases monotonically from $1 / 2$ to zero over the same range; in fact, for $r \gg M$ and $r \gg a,|\beta| \approx \sqrt{M / r}$.

It is interesting to mention here the behavior of $\beta$ for $a>M$. As before, $\beta$ is negative for counterrotating orbits, while for corotating orbits $\beta$ can be positive or negative. In fact, for a corotating orbit in this case $(a>M), \beta$ is positive for $r>a^{2} / M$, negative for $r<a^{2} / M$, and zero for $r=a^{2} / M$.

In Eq. (46), it is important to note the periodic motion in the radius: it oscillates between $r$ and $r+6 A \gamma^{2} \beta s^{*} / \rho^{2}$ with a proper period of $2 \pi /\left(\rho\left|\Omega_{\mathrm{K}}\right|\right)$. For the motion of the Earth about the Sun, $r \gg M$ and the Møller radius of the Earth is about $200 \mathrm{~cm}$, so that the amplitude of this periodic variation in the astronomical unit amounts to

$$
\frac{\delta r}{r} \approx \frac{6}{c}\left|\Omega_{\mathrm{K}}\right| s^{*} \sim 10^{-14},
$$

which is a few orders of magnitude too small to be measurable at present. In this connection, it is interesting to note that a secular increase of the astronomical unit, amounting to about $10^{3} \mathrm{~cm}$ per century, has been recently reported based on the analysis of radiometric data [26,27]. Furthermore, the polar motion about the equatorial plane as given by Eq. (47) consists of a superposition of two frequencies: $\left|\Omega_{\mathrm{K}}\right|$ and $\zeta\left|\Omega_{\mathrm{K}}\right|$. For $r \gg M \geq a$, this motion in the polar direction exhibits a beat phenomenon. That is, the "fast" harmonic oscillation at essentially the Keplerian frequency $\left|\Omega_{\mathrm{K}}\right|$ is modulated by a "slow" beat frequency $\approx \omega_{g} / 2$, where $\omega_{g}=3 G M\left|\Omega_{\mathrm{K}}\right| /\left(2 c^{2} r\right)$ is the "geodetic" (i.e., de Sitter-Fokker) precession frequency for the unperturbed orbit. The net amplitude (in radians) of the polar motion for $r \gg M \geq a$ is independent of $c$ and is given by $\tilde{s} / \sqrt{G M r}$. Nevertheless, this maximum amplitude is a relativistic effect and builds up over a "long" time scale; this circumstance is reminiscent of another phenomenon related to relativistic nutation [28]. For the Earth, the yearly spin-induced up and down polar motion about the ecliptic has a net amplitude of about $10^{6} \mathrm{~cm}$ and beat period of $10^{8}$ years; that is, the amplitude of the polar motion away from the ecliptic develops gradually and reaches a maximum of about 10 kilometers over a period of about $25 \times$ $10^{6}$ years.
It would be very interesting to search for these radial and polar motions in the strong-field regime close to black holes or in relativistic binary systems.

Let us now compare and contrast these analytic (but approximate) results with the numerical analysis of the exact equations in Sec. IV.

\section{NUMERICAL INTEGRATION OF MPD EQUATIONS}

To determine the domain of validity of our linear approximation scheme, it is interesting to make a numerical comparison of our results with the full MPD equations, which must be written in a form that is suitable for numerical integration. A direct comparison is possible once the reduced MPD equations are expressed in terms of proper time $\sigma$ as [29]

$$
\begin{aligned}
\frac{d P^{\alpha}}{d \sigma}=- & \Gamma_{\mu \nu}^{\alpha} P^{\mu} u_{s}^{\nu}+\frac{1}{2 m} R_{\beta \rho \sigma}^{\alpha} \epsilon_{\mu \nu}^{\rho \sigma} S^{\mu} P^{\nu} u_{s}^{\beta}, \\
\frac{d S^{\alpha}}{d \sigma}= & -\Gamma_{\mu \nu}^{\alpha} S^{\mu} u_{s}^{\nu} \\
& +\left(\frac{1}{2 m^{3}} R_{\gamma \beta \rho \sigma} \epsilon_{\mu \nu}^{\rho \sigma} S^{\mu} P^{\nu} S^{\gamma} u_{s}^{\beta}\right) P^{\alpha}
\end{aligned}
$$

$$
\frac{d x^{\alpha}}{d \sigma}=u_{s}^{\alpha}=\mathcal{N}\left(P^{\alpha}+\frac{1}{2} \frac{S^{\alpha \beta} R_{\beta \gamma \mu \nu} P^{\gamma} S^{\mu \nu}}{m^{2}+\frac{1}{4} R_{\mu \nu \rho \sigma} S^{\mu \nu} S^{\rho \sigma}}\right)
$$

where $\mathcal{N}=-u_{s}^{\alpha} P_{\alpha} / m^{2}$ is a normalization parameter such that $g_{\mu \nu} u_{s}^{\mu} u_{s}^{\nu}=-1$ throughout the spinning particle's motion.

We wish to integrate these equations numerically in Kerr spacetime with the same boundary conditions as in the linear approximation. Thus, we assume that at $\sigma=0$ the particle starts from $x^{\mu}(\sigma=0)=(0, r, \pi / 2,0)$ in BoyerLindquist coordinates. Moreover, we choose initial conditions for the linear momentum such that $P^{\mu}(\sigma=0)=$ $m u_{s}^{\mu}(\sigma=0)$, where

$$
u_{s}^{\mu}(\sigma=0)=\lambda^{\mu}{ }_{\hat{0}}
$$

is given by Eq. (19) and corresponds to a stable circular equatorial orbit around the Kerr source. The initial conditions for the components of the spin vector are given by

$$
S^{\mu}(\sigma=0)=\lambda_{\hat{\alpha}}^{\mu}(\sigma=0) S^{\hat{\alpha}} .
$$

Using Eqs. (20)-(22) and (33) with $S_{\hat{0}}=0$, we find 
$S^{\mu}(\sigma=0)=\left(\frac{m L \tilde{s}}{r A} \sin \varphi, m A \tilde{s} \cos \varphi,-\frac{m s^{*}}{r}, \frac{m E \tilde{s}}{r A} \sin \varphi\right)$

in Boyer-Lindquist coordinates. In this way, Eqs. (45)-(48) should provide the linear approximation to the full solution of Eqs. (51)-(53), since, as shown in Appendix A, $\tau=$ $\sigma+O\left(s^{2}\right)$. The results of the integration of the reduced MPD equations for the specific case of a prograde orbit with $m=10^{-2} M, \vartheta=\varphi=\pi / 4, a=0.5 M$, and $r=$
$10 M$ are presented in Figs. 1-3. Specifically, the spherical polar coordinates $(r, \theta, \phi)$ characterizing the orbit of the spinning particle in the standard Boyer-Lindquist coordinates are plotted versus proper time $\sigma$ in Figs. 1-3, respectively.

The results of the numerical integration of the reduced MPD equations confirm that Eqs. (45)-(48) provide the proper linear approximation to these equations. It is important to remark that these linear terms primarily exhibit the results of spin-orbit coupling (cf. Appendix B); that is, the rotation of the central source, though possibly impor-

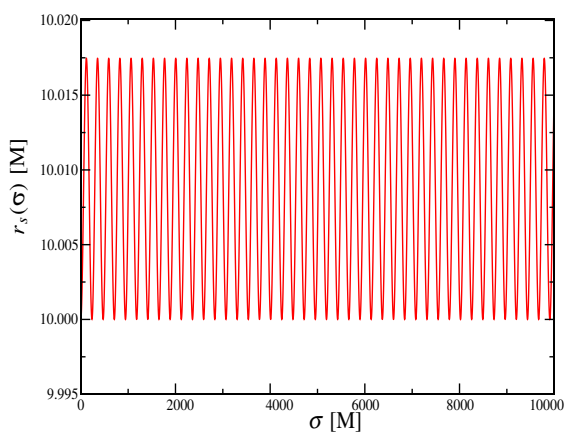

(b) $\quad s /(m r)=10^{-3}$ (Linear)
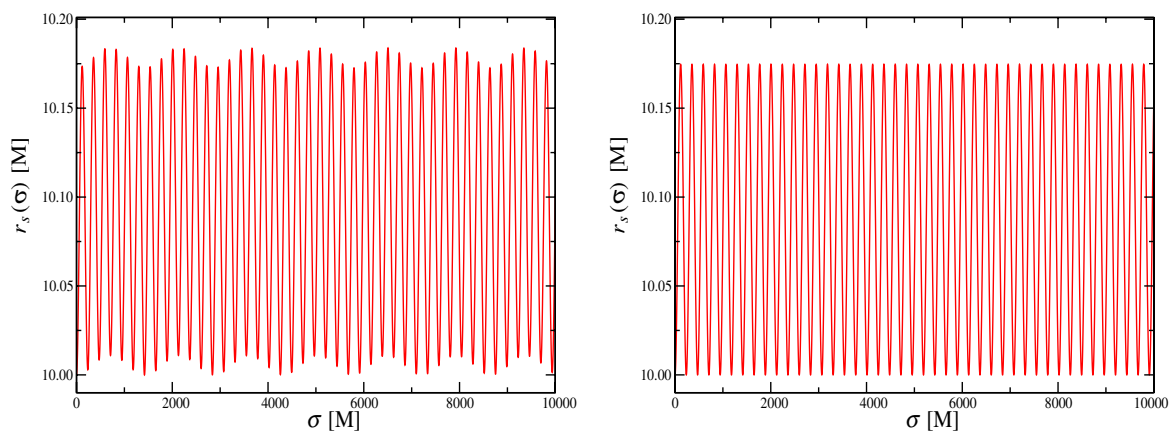

(d) $\quad s /(m r)=10^{-2}$ (Linear)

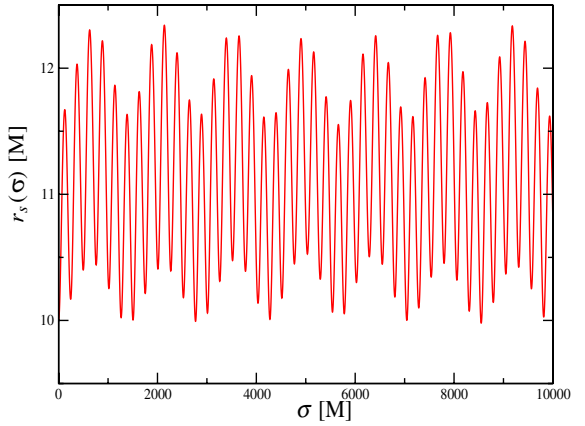

(e) $\quad s /(m r)=10^{-1}$ (MPD)

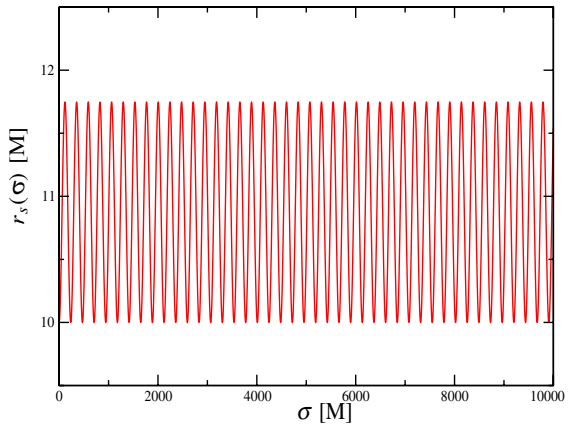

(f) $\quad s /(m r)=10^{-1}$ (Linear)

FIG. 1 (color online). The orbital radial coordinate $r_{s}(\sigma)$ for various choices of $s /(m r)$, where $m=10^{-2} M, r=10 M, \vartheta=\varphi=$ $\pi / 4$, and $a=0.50$. 

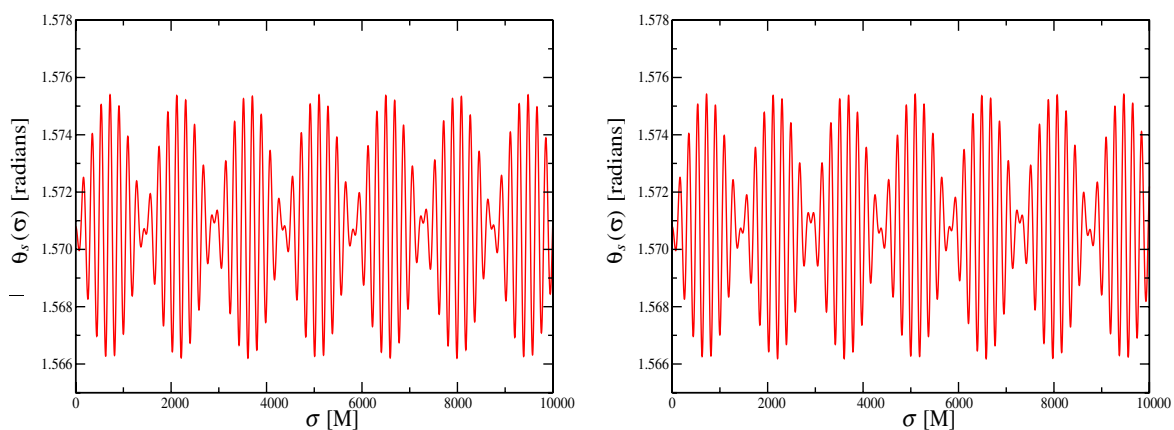

(a) $\quad s /(m r)=10^{-3}$ (MPD)

(b) $\quad s /(m r)=10^{-3}$ (Linear)
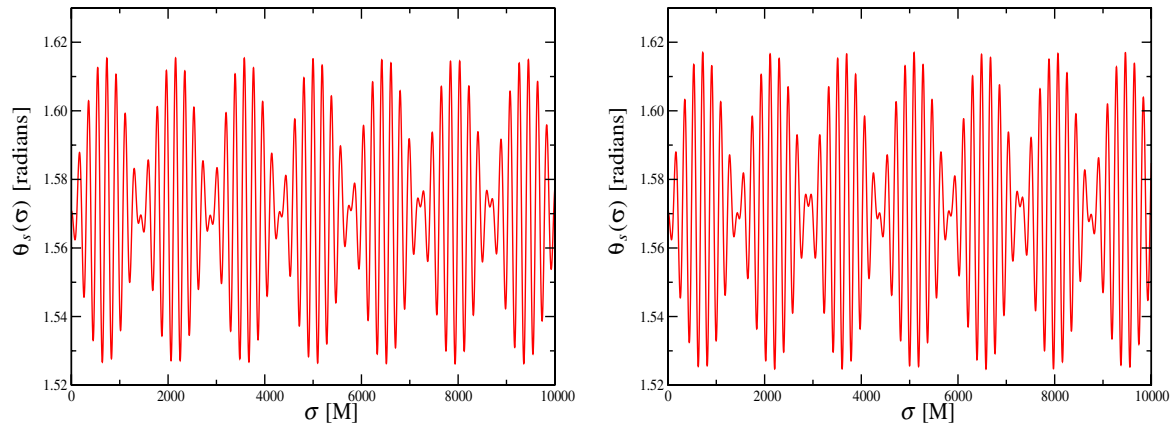

(c) $s /(m r)=10^{-2}$ (MPD)

(d) $\quad s /(m r)=10^{-2}$ (Linear)
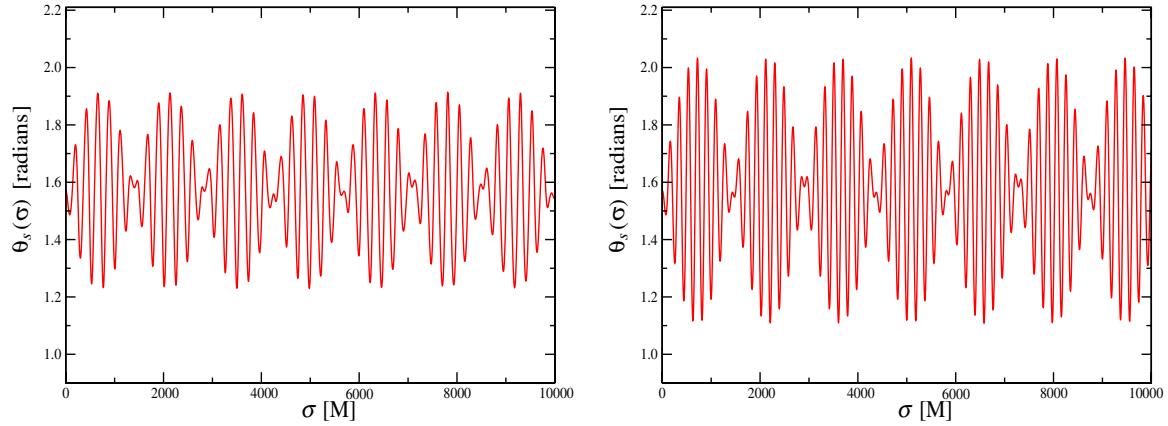

(e) $s /(m r)=10^{-1}$ (MPD)

(f) $\quad s /(m r)=10^{-1}$ (Linear)

FIG. 2 (color online). The orbital polar angle $\theta_{s}(\sigma)$ for various choices of $s /(m r)$, where $m=10^{-2} M, r=10 M, \vartheta=\varphi=\pi / 4$, and $a=0.50$

tant, is not crucial for the existence of the main phenomena associated with the motion of the particle in the first-order approximation. However, the rotation of the source could play a significant role in the difference between prograde and retrograde motion as in the clock effect discussed in Sec. V.

Beyond the linear approximation, it appears from Figs. 1-3 that for radial motion nonlinear spin terms introduce a complex beat phenomenon involving at least two periods. Of these, the higher frequency is about the same as the beat frequency in the case of polar motion. Furthermore, nonlinear spin effects are responsible for the reduction of the beat amplitude in the polar motion. On the other hand, the azimuthal motion appears to be essentially unaffected by the nonlinearities; therefore, only the graphs for the highest magnitude of spin considered here are presented in Fig. 3.

\section{GRAVITOMAGNETIC CLOCK EFFECT}

It follows from the results of the previous section that the analytic formulas involving $s /(m r)$ to first order are a good approximation to the exact solution of the reduced MPD equations; to go further, higher-order terms in spin must be 


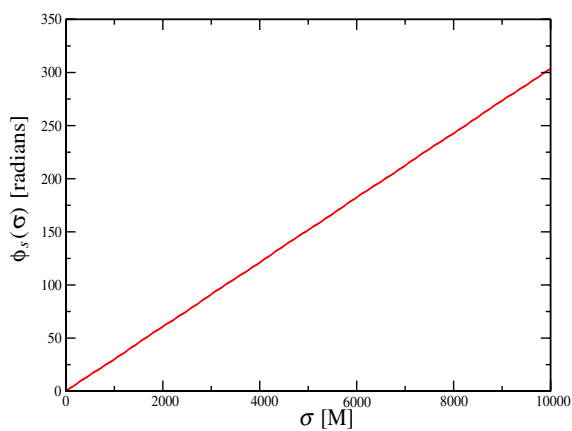

(a) $\quad s /(m r)=10^{-1}$ (MPD)

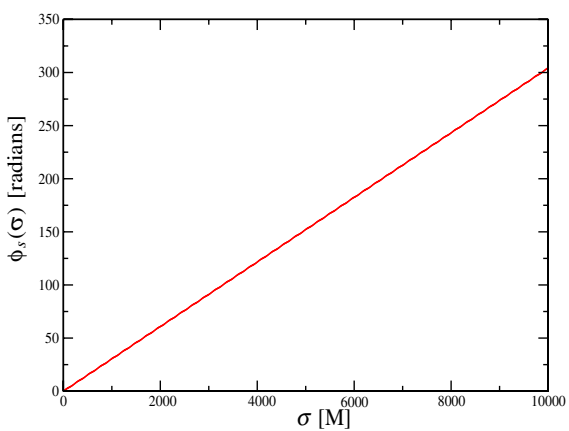

(b) $\quad s /(m r)=10^{-1}$ (Linear)

FIG. 3 (color online). The orbital azimuthal angle $\phi_{s}(\sigma)$, where $m=10^{-2} M, r=10 M, \vartheta=\varphi=\pi / 4$, and $a=0.50$.

taken into account. On the other hand, the analytic formulas can be safely applied within their theoretical limits of validity to illustrate a general feature of motion about a rotating source, namely, the gravitomagnetic clock effect [30-33]. In its simplest form, the effect involves a spinless "clock" on a circular orbit in the equatorial plane of a rotating source. The difference in the proper periods of two clocks on the same orbit but moving in opposite directions is given by $\tau_{+}-\tau_{-} \approx 4 \pi J /\left(M c^{2}\right)$ for $r \gg 2 G M / c^{2}$. Here $\tau_{+}\left(\tau_{-}\right)$is the proper period for the prograde (retrograde) motion. To lowest order, this remarkable effect is independent of Newton's constant of gravitation $G$ and the radius of the orbit. Moreover, the prograde motion is slower than the retrograde motion. For satellites in circular orbit about the Earth, $\tau_{+}-\tau_{-} \approx 10^{-7} \mathrm{~s}$; however, measuring this effect will not be simple [31].

The purpose of this section is to show that for a spinning clock, the analogous result to first order in $s /(m r)$ is

$$
\tau_{+}-\tau_{-} \approx 4 \pi\left[\frac{J}{M c^{2}}+6 \frac{s}{m c^{2}}(\hat{\boldsymbol{s}} \cdot \hat{\boldsymbol{J}})\right],
$$

based on azimuthal closure involving Eq. (48). This constitutes a generalization of previous results $[18,19,34]$ that involved only $\hat{\boldsymbol{s}} \cdot \hat{\boldsymbol{J}}= \pm 1$.

Imagine a spinning particle revolving around a Kerr source in accordance with Eqs. (45) -(48). After an integral number $p$ of complete orbits with $p \gg 1$, the change in $\phi_{\mathrm{s}}$ over a period of time $p \tau_{+}\left(p \tau_{-}\right)$for the corotating (counterrotating) clock is $2 \pi p(-2 \pi p)$. For $p \gg 1$, we can drop the $\sin (\rho \Theta)$ term in Eq. (48) in comparison with $\rho \Theta$; then, we find that the resulting expressions for $\tau_{+}$and $\tau_{-}$are such that the one for $\tau_{+}$changes over to the one for $\tau_{-}$if $a \rightarrow-a$ and $s \rightarrow-s$. To first order in $a / r$ and $s /(m r)$, we have

$$
\tau_{ \pm}=C T_{\mathrm{K}} \pm \frac{2 \pi}{C}\left(a+6 s^{*} \frac{B^{2} C^{2}}{D^{2}}\right),
$$

where

$$
B=\sqrt{1-\frac{2 M}{r}}, \quad C=\sqrt{1-\frac{3 M}{r}}, \quad D=\sqrt{1-\frac{6 M}{r}},
$$

$T_{\mathrm{K}}=2 \pi /\left|\Omega_{\mathrm{K}}\right|$ is the Keplerian period and $s^{*}$ given by Eq. (49) is proportional to the component of the specific spin of the clock along the axis of rotation of the source. Let us note that $\tau_{+}-\tau_{-}$computed from Eq. (58) for $r \gg$ $2 M$ would result in Eq. (57).

It is interesting to remark here that in the equatorial plane of Kerr spacetime,

$$
t_{+}-t_{-}=4 \pi a,
$$

which is another manifestation of the special gravitomagnetic temporal structure around the rotating source. Here $t_{+}$and $t_{-}$are the periods of geodesic motion around the same circular orbit according to the static inertial observers at spatial infinity. Substituting Eq. (58) in Eq. (45), we find along the same lines as before that the corresponding result for the motion of a spinning particle to first order in $s /(m r)$ and $a / r$ is $t_{ \pm}=T_{\mathrm{K}} \pm 2 \pi\left(a+6 s^{*} C^{2} / D^{2}\right)$, so that

$$
t_{+}-t_{-}=4 \pi\left(a+6 s^{*} \frac{C^{2}}{D^{2}}\right) .
$$

These results are in qualitative agreement with previous work in this direction $[18,19,34]$ that has been restricted to circular orbits for the motion of the spinning particle around the Kerr source with the corresponding limitation that the spin of the particle has to be parallel or antiparallel to the Kerr axis [15]. In this connection, it is important to remark here that Eqs. (45)-(48) explicitly forbid a circular equatorial orbit with $\cos \vartheta= \pm 1$. Thus, a direct quantitative comparison with previous work is not possible in this case. The contribution of spin to the clock effect is further elucidated in Appendix B.

\section{CONCLUSION}

We have developed in this paper a general first-order approximation scheme for the effect of spin on the motion 
of an extended spinning test particle in a gravitational field in accordance with the MPD equations that are based on the multipole expansion method. We have neglected the influence of quadrupole and higher moments on the motion. For the astrophysically interesting case of motion in the field of a central source, we must have $s /($ mcr $) \ll 1$, where $s /(m c)$ is the Møller radius of the extended test particle. Thus the influence of the spin of the particle on its motion is treated to linear order throughout. It turns out that in this approximation the spin is parallel transported along the nongeodesic path of the test particle. To illustrate our general approach, the motion of spinning test particles along nearly circular equatorial orbits in the exterior gravitational field of a Kerr source has been investigated in detail and the results of the first-order approximation scheme have been numerically compared with the corresponding solution of the full MPD equations. As expected, the results are essentially identical for $s /(\mathrm{mcr})$ sufficiently small compared to unity; moreover, we have described the deviations that appear when $s /(\mathrm{mcr})$ is not so small.

The astrophysical implications of our results have been briefly mentioned in Sec. III. For the motion of the Earth about the Sun, for instance, the spin-induced deviations from Keplerian motion are too small to be observationally significant at present; however, the spin-dependent terms could become important in binary pulsars [35,36]. Furthermore, we have elucidated the gravitomagnetic clock effect for spinning particles in accordance with our firstorder approximation method.

\section{ACKNOWLEDGMENTS}

D. S. wishes to thank Nader Mobed of the University of Regina for financial support via a NSERC Discovery Grant.

\section{APPENDIX A: MPD EQUATIONS IN FERMI NORMAL COORDINATES}

Consider a Fermi normal coordinate system that is constructed about the reference geodesic $x^{\mu}(\tau)$ and is based upon the parallel-propagated orthonormal frame $\lambda^{\mu}{ }_{\alpha}$. Specifically, along the worldline of the spinning particle $x_{s}^{\mu}$, let us imagine an event $Q_{s}$ with Fermi coordinates $X^{\mu}=(T, X)$; then, there exists a unique spacelike geodesic of proper length $l$ that connects $Q_{s}$ orthogonally to the reference geodesic $x^{\mu}$ at the event $Q_{0}$ with proper time $\tau$ such that

$$
T=\tau, \quad X_{i}=\ln _{\mu} \lambda_{\hat{i}}^{\mu},
$$

where $n_{\mu}=\left(d x^{\mu} / d l\right)_{Q_{0}}$ is the unit vector tangent to the unique spacelike geodesic at $Q_{0}$ such that $n_{\mu} u^{\mu}=0$. Thus the spinless reference particle following $x^{\mu}(\tau)$ permanently occupies the spatial origin of this Fermi coordinate system and has Fermi coordinates $(T, \mathbf{0})$, where $T=\tau$. The spacetime metric in Fermi coordinates is given by

$$
\begin{gathered}
g_{00}=-1-R_{\hat{0} \hat{\imath} \hat{\jmath}}(T) X^{i} X^{j}+\cdots, \\
g_{0 i}=-\frac{2}{3} R_{\hat{0} \hat{\jmath} \hat{\imath} \hat{k}}(T) X^{j} X^{k}+\cdots, \\
g_{i j}=\delta_{i j}-\frac{1}{3} R_{\hat{\imath} \hat{k} \hat{\jmath}}(T) X^{k} X^{l}+\cdots,
\end{gathered}
$$

where $R_{\hat{\alpha} \hat{\beta} \hat{\gamma} \hat{\delta}}$, given by the projection of the Riemann curvature tensor on the tetrad frame of the spinless reference particle, is in fact the Riemann tensor in the Fermi coordinate system evaluated at its spatial origin. The Fermi coordinate system is admissible within a cylindrical spacetime region around the worldline of the reference geodesic such that $|X|<\mathcal{R}_{a}(T)$, where $\mathcal{R}_{a}$ is a certain minimum radius of curvature of spacetime.

The Mathisson-Papapetrou-Dixon equations of motion of the spinning particle within the framework of our firstorder approximation scheme (based on $s \ll m r$ ) can be expressed in the Fermi coordinate system as

$$
\begin{gathered}
\frac{D U^{\mu}}{d \sigma}=\mathcal{A}^{\mu}, \quad \mathcal{A}^{\mu}=-\frac{1}{2 m} R_{\gamma \alpha \beta}^{\mu} U^{\gamma} S^{\alpha \beta}, \\
\frac{D S^{\mu \nu}}{d \sigma}=0, \quad S_{\mu \nu} U^{\nu}=0,
\end{gathered}
$$

where $U^{\mu}=d X^{\mu} / d \sigma$ is the four-velocity of the spinning particle. Let us write this as

$$
U^{\mu}=\Gamma(1, \boldsymbol{V}), \quad \Gamma=\frac{d T}{d \sigma}, \quad \boldsymbol{V}=\frac{d \boldsymbol{X}}{d T} .
$$

Then,

$$
\Gamma^{-2}=-g_{00}-2 g_{0 i} V^{i}-g_{i j} V^{i} V^{j}>0,
$$

since $U^{\mu}$ is a timelike unit vector. To characterize the order of various quantities in accordance with our perturbation method, it is convenient to write

$$
\boldsymbol{X}(T)=O(s), \quad \boldsymbol{V}(T)=O(s), \quad \frac{1}{\Gamma^{2}}=1+O\left(s^{2}\right),
$$

and so on; in particular, it follows that $\sigma=T+O\left(s^{2}\right)$. We have already discussed the consequences of Eq. (A6) in Sec. II; therefore, we concentrate here on Eq. (A5). We find $[6,37]$

$$
\frac{d^{2} X^{i}}{d T^{2}}+\left(\Gamma_{\alpha \beta}^{i}-\Gamma_{\alpha \beta}^{0} V^{i}\right) \frac{d X^{\alpha}}{d T} \frac{d X^{\beta}}{d T}=\frac{1}{\Gamma^{2}}\left(\mathcal{A}^{i}-\mathcal{A}^{0} V^{i}\right),
$$

where

$$
\mathcal{A}^{0}=0, \quad \mathcal{A}^{i}=\frac{1}{2 m} R_{\hat{0} \hat{\imath} \hat{\jmath} \hat{k}}(T) S^{\hat{\jmath} \hat{k}} .
$$

We note that the Mathisson-Papapetrou acceleration is given by 


$$
\mathcal{A}^{i}=\frac{1}{m} \mathcal{H}_{i j} S^{\hat{\jmath}}
$$

based on the definitions introduced in Sec. II. Moreover, of the connection coefficients in Eq. (A10) only $\Gamma_{00}^{i}=\mathcal{E}_{i j} X^{j}$ makes a non-negligible contribution. Thus taking due account of our first-order approximation scheme, we find that Eqs. (A10)-(A12) reduce to

$$
\frac{d^{2} X^{i}}{d T^{2}}+\mathcal{E}_{i j} X^{j}=\mathcal{A}^{i}
$$

with the boundary conditions that $\boldsymbol{X}=\mathbf{0}$ and $\boldsymbol{V}=\mathbf{0}$ at $T=$ 0 . We now turn to the general solution of Eq. (A13), which may be expressed as

$$
\frac{d \Psi}{d T}=\left(\begin{array}{cc}
0 & I \\
-\mathcal{E} & 0
\end{array}\right) \Psi+\chi,
$$

where $I$ is the $3 \times 3$ unit matrix and

$$
\Psi=\left(\begin{array}{l}
\boldsymbol{X} \\
\boldsymbol{V}
\end{array}\right), \quad \chi=\left(\begin{array}{c}
0 \\
\mathcal{A}
\end{array}\right) .
$$

Let $\psi$ be a general solution of the homogeneous (Jacobi) system with $\chi=\mathbf{0}$; then,

$$
\psi=\sum_{i=1}^{6} c_{i} \psi_{i},
$$

where the $c_{i}, i=1,2, \cdots, 6$, are arbitrary constants that physically correspond to the initial position and velocity of a free particle following the Jacobi equation and $\psi_{i}, i=$ $1,2, \cdots, 6$, form a fundamental set of solutions of the Jacobi system. According to the method of variation of parameters [8], we seek a solution of the inhomogeneous Eq. (A14) by assuming that $c_{i} \rightarrow C_{i}(T)$, i.e., we let

$$
\Psi=\sum_{i=1}^{6} C_{i}(T) \psi_{i} .
$$

Substitution of Eq. (A17) in Eq. (A14) results in

$$
\sum_{i=1}^{6} \frac{d C_{i}}{d T} \psi_{i}=\chi .
$$

Consider a $6 \times 6$ matrix $\Phi$ that is the fundamental matrix solution of the homogeneous Jacobi system and contains $\psi_{1}, \psi_{2}, \cdots, \psi_{6}$ as its column vectors. Then $\Psi=\Phi \mathcal{C}$, where $\mathcal{C}$ is a column vector with $C_{1}, C_{2}, \cdots, C_{6}$ as its elements, and Eq. (A18) can be written as

$$
\frac{d \mathcal{C}}{d T}=\Phi^{-1} \chi
$$

With the initial conditions that $\Psi=\mathcal{C}=0$ at $T=0$, the solution of Eq. (A14) is

$$
\Psi(T)=\Phi(T) \int_{0}^{T} \Phi^{-1}\left(T^{\prime}\right) \chi\left(T^{\prime}\right) d T^{\prime} .
$$

In principle, the trajectory of the spinning particle can thus be determined in the Fermi coordinate system. To express this trajectory in terms of the original background coordinate system, it is in general necessary to have the explicit coordinate transformation between the two systems of coordinates. This turns out to be possible only in very special situations [38,39]. However, our first-order approximation scheme makes it possible to proceed as follows. The deviation vector $\delta x^{\mu}$ may be written as $\delta x^{\mu} \approx l n^{\mu}$, since $l \ll \mathcal{R}_{a}$. Therefore, regarding $\delta x^{\mu}$ as a vector field along the reference geodesic, we can write $\delta x^{\mu}=X^{i}(\tau) \lambda^{\mu}{ }_{\hat{\imath}}$, where the Fermi temporal coordinate in the solution of Eq. (A13), namely, $\boldsymbol{X}(T)$, has been replaced by $\tau$. It follows that $x_{s}^{\mu}=x^{\mu}+X^{i} \lambda^{\mu}{ }_{\hat{i}}$; in this way, we recover Eqs. (6) and (12) of Sec. II.

\section{APPENDIX B: HEURISTIC INTERPRETATION OF THE CLOCK EFFECT FOR SPINNING PARTICLES}

The purpose of this appendix is to provide an intuitive physical understanding for the appearance of terms proportional to $s^{*}$ in the discussion of the gravitomagnetic clock effect in Sec. V. The existence of such a term was first pointed out in Ref. [18] and has been further studied in $[19,34]$. The approach adopted here is based on the gravitoelectromagnetic (GEM) analogy; we follow here the basic conventions of Ref. [40].

Consider a spinning particle in orbit about a central nonrotating source. In the rest frame of the particle, the central source revolves around the particle and this motion generates a gravitomagnetic field. The resulting spingravitomagnetic field interaction, just as in the electromagnetically analogous case of the hydrogen atom, must be corrected by taking due account of Thomas precession, which in the gravitational case becomes the de SitterFokker (geodetic) precession. The combined interaction due to these terms is of the spin-orbit coupling form [36]. Specifically, in the rest frame of the spinning particle, the gravitomagnetic field $\boldsymbol{B}_{g}$ is proportional to $-(\boldsymbol{v} / c) \times \boldsymbol{E}_{g}$, where $\boldsymbol{E}_{g}=G M \boldsymbol{r} / r^{3}$. The corresponding contribution to the Hamiltonian, i.e. the analogue of $-\boldsymbol{\mu} \cdot \boldsymbol{B}$, would be [40]

$$
\frac{1}{c} \boldsymbol{s} \cdot \boldsymbol{B}_{g}=\xi_{0}\left(\frac{G M}{m c^{2} r^{3}}\right) \boldsymbol{s} \cdot \boldsymbol{L},
$$

where $\boldsymbol{L}=m \boldsymbol{r} \times \boldsymbol{v}$ and $\xi_{0}$ is a numerical factor that is expected to be of order unity. Furthermore, the geodetic precession frequency is given by

$$
\boldsymbol{\omega}_{g}=\frac{3}{2}\left(\frac{G M}{m c^{2} r^{3}}\right) \boldsymbol{L},
$$

hence the corresponding contribution to the Hamiltonian would be

$$
\boldsymbol{s} \cdot \boldsymbol{\omega}_{g}=\frac{3}{2}\left(\frac{G M}{m c^{2} r^{3}}\right) \boldsymbol{s} \cdot \boldsymbol{L} .
$$


Therefore, the net Hamiltonian for the motion of the particle may be taken to be of the form

$$
H=\frac{p^{2}}{2 m}-\frac{G M m}{r}+\xi\left(\frac{G M}{m c^{2} r^{3}}\right) \boldsymbol{s} \cdot \boldsymbol{L}
$$

in this simple GEM model. Here,

$$
\xi=\xi_{0}+\frac{3}{2}
$$

In Eq. (B4), the spin-orbit term is a small relativistic correction to the Newtonian dynamics and turns out to be the source of the spin-dependence of the clock effect [34].

It follows from Hamilton's equations of motion for Eq. (B4) that

$$
\begin{aligned}
& \frac{d \boldsymbol{r}}{d t}=\frac{\boldsymbol{p}}{m}+\xi\left(\frac{G M}{m c^{2} r^{3}}\right) \boldsymbol{s} \times \boldsymbol{r}, \\
\frac{d \boldsymbol{p}}{d t}= & -\left(\frac{G M m}{r^{3}}\right) \boldsymbol{r}+3 \xi\left(\frac{G M}{m c^{2} r^{5}}\right)(\boldsymbol{s} \cdot \boldsymbol{L}) \boldsymbol{r} \\
& -\xi\left(\frac{G M}{m c^{2} r^{3}}\right) \boldsymbol{p} \times \boldsymbol{s} .
\end{aligned}
$$

Thus the canonical momentum can be expressed as

$$
\boldsymbol{p}=m \boldsymbol{v}+\xi\left(\frac{G M}{c^{2} r^{3}}\right) \boldsymbol{r} \times \boldsymbol{s}
$$

and the equation of motion of the spinning particle is then given by the substitution of Eq. (B8) in Eq. (B7). To first order in the spin-dependent contribution, the result is

$$
\frac{d^{2} \boldsymbol{r}}{d t^{2}}+\left(\frac{G M}{r^{3}}\right) \boldsymbol{r}=\frac{\boldsymbol{F}}{m},
$$

where $\boldsymbol{F}$ is the spin-dependent force given by

$\boldsymbol{F}=\xi\left(\frac{G M}{c^{2} r^{3}}\right)\left\{\frac{3}{r} \frac{d r}{d t}(\boldsymbol{r} \times \boldsymbol{s})-2(\boldsymbol{v} \times \boldsymbol{s})+\frac{3}{r^{2}}[(\boldsymbol{r} \times \boldsymbol{v}) \cdot \boldsymbol{s}] \boldsymbol{r}\right\}$.

Consider an initially circular Keplerian orbit of radius $r$ in the equatorial $(x, y)$ plane. At $t=0$, the orbit is perturbed by the spin-dependent force

$$
\boldsymbol{F}=\xi \Omega_{\mathrm{K}}\left(\frac{G M}{c^{2} r^{3}}\right)[(s \cos \vartheta) \boldsymbol{r}+2(\boldsymbol{s} \cdot \boldsymbol{r}) \hat{z}],
$$

where $s=(s, \vartheta, \varphi)$ in spherical polar coordinates and

$$
\boldsymbol{r}=r\left[\left(\cos \Omega_{\mathrm{K}} t\right) \hat{\boldsymbol{x}}+\left(\sin \Omega_{\mathrm{K}} t\right) \hat{\boldsymbol{y}}\right] .
$$

Let us write the equation of the perturbed orbit in cylindrical coordinates as

$$
r_{s}=r(1+f), \quad \phi_{s}=\Omega_{\mathrm{K}} t+q, \quad z_{s}=r h,
$$

where $f, q$, and $h$ denote spin-dependent perturbations. Imposing the boundary conditions that at $t=0$,

$$
f=q=h=0, \quad \frac{d f}{d t}=\frac{d q}{d t}=\frac{d h}{d t}=0,
$$

we find, using the method developed in Ref. [41], that

$$
r_{s}=r+\xi \sqrt{\frac{G M}{c^{2} r}} s^{*}\left(1-\cos \Omega_{\mathrm{K}} t\right),
$$

$$
\begin{gathered}
\phi_{s}=\Omega_{\mathrm{K}} t-\frac{2}{c} \xi \Omega_{\mathrm{K}} s^{*}\left(\Omega_{\mathrm{K}} t-\sin \Omega_{\mathrm{K}} t\right), \\
z_{s}=\xi \sqrt{\frac{G M}{c^{2} r}} \tilde{s}\left[\sin \left(\Omega_{\mathrm{K}} t-\varphi\right) \Omega_{\mathrm{K}} t+\sin \varphi \sin \Omega_{\mathrm{K}} t\right] .
\end{gathered}
$$

It is interesting to compare Eqs. (B15)-(B17) with Eqs. (45)-(48); in fact, to lowest relativistic order, we find from the latter equations the same results as Eqs. (B15) and (B16) with $\xi=3$. Moreover, with $\zeta \Theta \approx$ $\Omega_{\mathrm{K}} t, \zeta \approx 1+(3 / 2) \beta^{2}$, and $\beta^{2} \approx G M /\left(c^{2} r\right)$, Eq. (47) implies that

$$
\theta_{s} \approx \frac{\pi}{2}-\frac{3}{2} \sqrt{\frac{G M}{c^{2} r}} \frac{\tilde{s}}{r}\left[\sin \left(\Omega_{\mathrm{K}} t-\varphi\right) \Omega_{\mathrm{K}} t+\sin \varphi \sin \Omega_{\mathrm{K}} t\right] .
$$

We note that $z_{s}=r_{s} \cos \vartheta_{s}$, so that one obtains from Eqs. (45)-(48) that

$$
z_{s} \approx \frac{3}{2} \sqrt{\frac{G M}{c^{2} r}} \tilde{S}\left[\sin \left(\Omega_{\mathrm{K}} t-\varphi\right) \Omega_{\mathrm{K}} t+\sin \varphi \sin \Omega_{\mathrm{K}} t\right],
$$

which is smaller by a numerical factor of $1 / 2$ than the expression given by Eq. (B17) with $\xi=3$. Nevertheless, it is rather remarkable that our simple model, based on the GEM analogy, predicts the main qualitative features of the perturbed orbit. In particular, it follows from Eq. (B16) that

$$
t_{ \pm}=T_{K} \pm 4 \pi \xi s^{*}
$$

hence, for $\xi=3$ the spin part of the clock effect is recovered.

We have thus far ignored the proper rotation of the central source. The rotation of the source would generate a gravitomagnetic field and the spin of the test mass naturally couples to this gravitomagnetic field as described in detail in Ref. [6]. It turns out, however, that the corresponding dominant "hyperfine" coupling term is independent of the sense of the orbit and hence does not contribute to the main spin-dependent gravitomagnetic clock effect under consideration here.

Finally, a remark is in order here regarding the fact that with $\xi=3$ and $\xi=\xi_{0}+3 / 2$, we have $\xi_{0}=3 / 2$, so that the $\boldsymbol{s} \cdot \boldsymbol{B}_{g} / c$ and geodetic terms, given, respectively, by Eqs. (B1) and (B3), contribute equally to the net spin-orbit coupling term in the classical Hamiltonian (B4). As noted in Ref. [34], a similar result has been obtained in the treatment of a Dirac particle in the Schwarzschild field [42-45]. 
[1] W. G. Dixon, Phil. Trans. R. Soc. A 277, 59 (1974).

[2] J.L. Synge, Relativity: The General Theory (NorthHolland, Amsterdam, 1960).

[3] W. G. Dixon, Proc. R. Soc. A 314, 499 (1970); 319, 509 (1970); Gen. Relativ. Gravit. 4, 199 (1973).

[4] M. Mathisson, Acta Phys. Pol. 6, 163 (1937).

[5] A. Papapetrou, Proc. R. Soc. London 209, 248 (1951).

[6] C. Chicone, B. Mashhoon, and B. Punsly, Phys. Lett. A 343, 1 (2005).

[7] J. Ehlers and R. Geroch, Ann. Phys. (N.Y.) 309, 232 (2004).

[8] C. Chicone, Ordinary Differential Equations with Applications (Springer-Verlag, Leipzig, 2006), 2nd ed.

[9] H. Quevedo and B. Mashhoon, Phys. Rev. D 43, 3902 (1991); V.S. Manko, E. W. Mielke, and J. D. SanabriaGómez, Phys. Rev. D 61, 081501(R) (2000).

[10] C. W. Misner, K. S. Thorne, and J. A. Wheeler, Gravitation (W. H. Freeman and Company, New York, 1973).

[11] S. Chandrasekhar, The Mathematical Theory of Black Holes (Oxford University, New York, 1992).

[12] B. Mashhoon, J. Math. Phys. (N.Y.) 12, 1075 (1971); R. Wald, Phys. Rev. D 6, 406 (1972).

[13] K. P. Tod, F. de Felice, and M. Calvani, Nuovo Cimento B 34, 365 (1976).

[14] E. Schmutzer and J. Plebanski, Fortschr. Phys. 25, 37 (1977).

[15] M. A. Abramowicz and M. Calvani, Mon. Not. R. Astron. Soc. 189, 621 (1979).

[16] R. Plyatsko, Phys. Rev. D 58, 084031 (1998); R. Plyatsko and O. Bilaniuk, Classical Quantum Gravity 18, 5187 (2001); R. Plyatsko, Classical Quantum Gravity 22, 1545 (2005).

[17] O. Semerák, Mon. Not. R. Astron. Soc. 308, 863 (1999).

[18] S. B. Faruque, Phys. Lett. A 327, 95 (2004).

[19] D. Bini, F. de Felice, and A. Geralico, Classical Quantum Gravity 21, 5441 (2004); D. Bini, C. Cherubini, A. Geralico, and B. Mashhoon, Classical Quantum Gravity 22, 709 (2005).

[20] T. Tanaka, Y. Mino, M. Sasaki, and M. Shibata, Phys. Rev. D 54, 3762 (1996).

[21] S. Suzuki and K. I. Maeda, Phys. Rev. D 58, 023005 (1998).

[22] M. Mohseni, R.W. Tucker, and C. Wang, Classical Quantum Gravity 18, 3007 (2001); S. Kessari, D. Singh, R. W. Tucker, and C. Wang, Classical Quantum Gravity 19, 4943 (2002).

[23] M. D. Hartl, Phys. Rev. D 67, 024005 (2003); 67, 104023 (2003).

[24] C. Chicone and B. Mashhoon, Classical Quantum Gravity 23, 4021 (2006).

[25] J.-A. Marck, Proc. R. Soc. A 385, 431 (1983).
[26] E. M. Standish, in Proceedings of the IAU Colloquium No. 196, edited by D. W. Kurtz (Cambridge University, Cambridge, England, 2005), p. 163; G. A. Krasinsky and V. A. Brumberg, Celest. Mech. Dyn. Astron. 90, 267 (2004).

[27] C. Lämmerzahl, O. Preuss, and H. Dittus, gr-qc/0604052.

[28] B. Mashhoon and D. S. Theiss, Phys. Rev. Lett. 49, 1542 (1982); Nuovo Cimento B 106, 545 (1991); Lect. Notes Phys. 562, 310 (2001).

[29] D. Singh, Phys. Rev. D 72, 084033 (2005).

[30] J.M. Cohen and B. Mashhoon, Phys. Lett. A 181, 353 (1993); B. Mashhoon, L. Iorio, and H. Lichtenegger, Phys. Lett. A 292, 49 (2001).

[31] B. Mashhoon, F. Gronwald, and D. S. Theiss, Ann. Phys. (Leipzig) 8, 135 (1999); B. Mashhoon, F. Gronwald, and H. I. M. Lichtenegger, Lect. Notes Phys. 562, 83 (2001); L. Iorio and H.I.M. Lichtenegger, Classical Quantum Gravity 22, 119 (2005); H. I. M. Lichtenegger, L. Iorio, and B. Mashhoon, Ann. Phys. (Leipzig) 15, 868 (2006).

[32] D. Bini, R.T. Jantzen, and B. Mashhoon, Classical Quantum Gravity 18, 653 (2001); 19, 17 (2002).

[33] D. Bini, F. de Felice, and A. Geralico, Classical Quantum Gravity 21, 5427 (2004); D. Bini, F. de Felice, A. Geralico, and R. T. Jantzen, Classical Quantum Gravity 22, 2947 (2005); D. Bini, A. Geralico, and F. de Felice, Int. J. Mod. Phys. D 14, 1793 (2005); D. Bini, F. de Felice, A. Geralico, and R. T. Jantzen, Classical Quantum Gravity 23, 3287 (2006).

[34] S. B. Faruque, Phys. Lett. A 359, 252 (2006).

[35] L. Iorio, Int. J. Mod. Phys. D 12, 35 (2003).

[36] B. M. Barker and R. F. O'Connell, Phys. Rev. D 12, 329 (1975); R. F. O'Connell, Phys. Rev. Lett. 93, 081103 (2004).

[37] C. Chicone and B. Mashhoon, Classical Quantum Gravity 22, 195 (2005).

[38] D. Bini, A. Geralico, and R. T. Jantzen, Classical Quantum Gravity 22, 4729 (2005).

[39] C. Chicone and B. Mashhoon, Phys. Rev. D 74, 064019 (2006).

[40] B. Mashhoon, gr-qc/0311030.

[41] B. Mashhoon, Astrophys. J. 223, 285 (1978).

[42] T.-Y. Lee, Phys. Lett. A 291, 1 (2001).

[43] F. W. Hehl and W.-T. Ni, Phys. Rev. D 42, 2045 (1990).

[44] J. Audretsch, F. W. Hehl, and C. Lämmerzahl, Lect. Notes Phys. 410, 368 (1992); F. W. Hehl, J. D. McCrea, E. W. Mielke, and Y. Ne'eman, Phys. Rep. 258, 1 (1995); F. W. Hehl, J. Lemke, and E.W. Mielke, in Geometry and Theoretical Physics, edited by J. Debrus and A.C. Hirshfeld (Springer-Verlag, Berlin, 1991), p. 56.

[45] K. Varjú and L. H. Ryder, Phys. Lett. A 250, 263 (1998). 